\title{
Gout mimicking spondyloarthritis: case report and literature review
}

\author{
Wenji Chen' \\ Yanyan Wang ${ }^{2}$ \\ Yan $\mathrm{Li}^{2}$ \\ Zheng Zhao ${ }^{2}$ \\ Lixia Feng ${ }^{2}$ \\ Jian $\mathrm{Zhu}^{2}$ \\ Jianglin Zhang ${ }^{2}$ \\ Feng Huang ${ }^{2}$ \\ 'Department of Rheumatology, \\ Hainan Branch of Chinese People's \\ Liberation Army General Hospital, \\ Sanya, ${ }^{2}$ Department of Rheumatology, \\ Chinese People's Liberation Army \\ General Hospital, Beijing, People's \\ Republic of China
}

This article was published in the following Dove Press journal: Journal of Pain Research

29 June 2017

Number of times this article has been viewed

\begin{abstract}
Gout is clinically characterized by episodes of monoarthritis, which not only typically affects the peripheral joints but also occasionally affect the axial joint, such as the sacroiliac joint (SIJ), and often mimics spondyloarthritis (SpA). Two cases of gout mimicking SpA are presented in the current paper. One patient was a 32-year-old man with a history of asymmetrical oligoarthritis of ankle and metatarsophalangeal joints (MTPJ). He had left gluteal pain for 2 weeks. Computed tomography (CT) and magnetic resonance imaging (MRI) revealed the bone erosion of the left SIJ. T1-weighted MRI showed hypointense T1 and hyperintense T2 signals of the left SIJ. The other patient was a 24-year-old man with left back pain and hip pain for 4 months and intermittent fever for 3 months. He had a history of gout for 3 years. Both patients underwent CT-guided sacroiliac biopsy, and monosodium urate (MSU) crystals were shown by polarized microscopy. Gout can often mimic SpA and seldomly affects the SIJ. Thus, its correct diagnosis and adequate therapy can halt the development of such damaging complications.
\end{abstract} Keywords: gout, crystal arthropathy, sacroiliac joint, radiography

\section{Introduction}

Gout not only typically affects the peripheral joints but also occasionally affects the axial joint, such as the sacroiliac joint (SIJ). ${ }^{1}$ It manifests as low back or hip pain, and these manifestations are the first presentation of gout in many patients. Sometimes gout in the SIJ mimics spondyloarthritis (SpA), although X-rays, computed tomography (CT) and magnetic resonance imaging (MRI) scans are nonspecific and their results vary. $^{2-4}$ Histopathological, cytological and crystal analyses are the diagnostic gold standard. Two patients with poorly controlled gout that affected the SIJs and resulted in low back and hip pain are presented in the current paper. Written informed consents were provided by the two patients to have their case details and any accompanying images published.

\section{Case presentation Case I}

The first case was a 32-year-old man with intermittent joint swelling and pain over 6 years, which aggravated 2 months ago. The patient had also experienced left gluteal pain for 2 weeks. The first metatarsophalangeal joint (MTPJ) of the left foot was affected at the onset, and the pain gradually involved all MTPJs, both ankles and knees, left elbow and tarsal joints of both feet. The patient had a high purine diet history before joint swelling and pain in the early period of this disease, each episode involving one to two joints,
Correspondence: Yanyan Wang Department of Rheumatology, Chinese People's Liberation Army General Hospital, Fuxing Road 28, Beijing 100853 , People's Republic of China Tel +86 I38 I0820453

Email wangyanyan1990@163.com 
and intermission gradually reduced from 6 months to 1 week. His serum urate levels were elevated, but he did not accept regular treatment. Approximately 2 years ago, the patient took dexamethasone for joint swelling and pain, although the specific dosage was unknown. He had experienced right knee joint swelling and pain without any significant predisposing factor 2 months earlier, and the symptoms were relieved after intra-articular betamethasone injection. But, joint swelling and pain involving both ankles, tarsal joints and MTPJs reappeared 1 week after discontinuing treatment. The pain in the left gluteal region occurred 2 weeks before the current admission, and was sharp when changing position, but was unclear at rest and night. There was no infection of urinary and intestinal tracts before hip pain. The patient had no history of psoriasis and iritis. Lumbar disk herniation was diagnosed 7 years earlier because of low back pain with left lower limb radiating pain. Physical examination showed the following: obesity, a grain size of tophi visible on the edge of the right ear, right knee joint tenderness without swelling, double ankle joint tenderness with slight swelling and tenderness of all MTPJs of the left foot and tarsal joints of both feet, but no tenderness of the SIJ. Laboratory tests showed the following: serum uric acid (UA) $564.2 \mu \mathrm{mol} / \mathrm{L}$, triglycerides (TGs) $2.12 \mathrm{mmol} / \mathrm{L}$, erythrocyte sedimentation rate (ESR) $32 \mathrm{~mm} / \mathrm{h}$, negative human leukocyte antigen (HLA)-B27 and rheumatoid factor. CT of the SIJ showed bone erosion of the left SIJ (Figure 1). MRI of the SIJ showed the unilateral SIJs of the left articular surface with patchy hypointense T1 and hyperintense T2 signals. Diffusion-weighted imaging (DWI) showed the hyperintense signal of the left SIJ with edge blurring, mainly indicating the sacroiliitis (Figure 2). He underwent CT-guided percutaneous

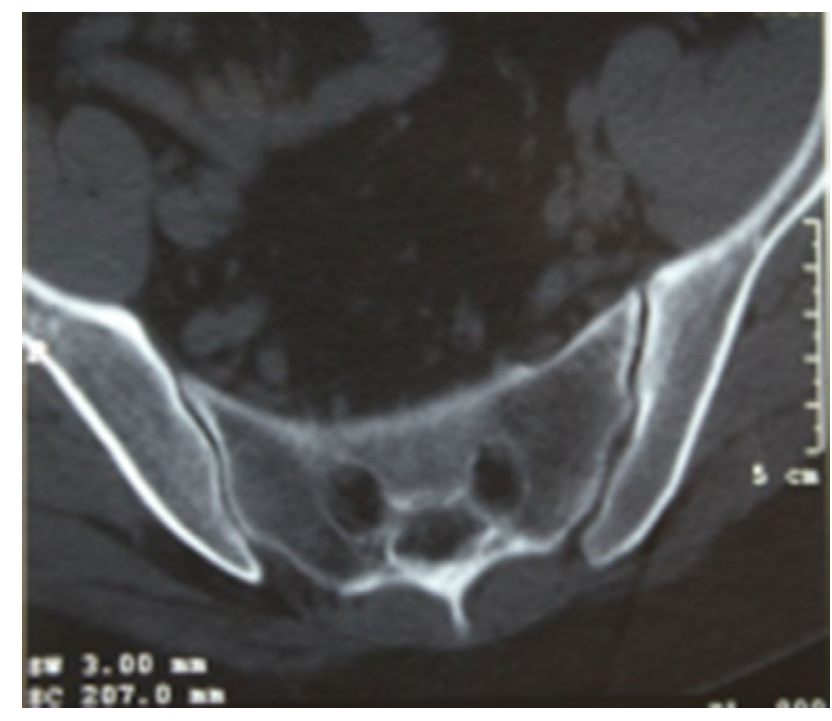

Figure I CT of the SIJ $(I 20 \mathrm{kV}, 280 \mathrm{~mA})$ shows bone erosion of the left SIJ. Abbreviations: CT, computed tomography; SIJ, sacroiliac joint. biopsy of the left SIJ, and monosodium urate (MSU) crystals were shown by polarized microscopy (Figure 3A), although histopathology of the puncture objects did not indicate tophus formation (Figure 3B). Therefore, the clinical diagnosis of gouty arthritis was correct. Loxoprofen sodium tablets were given to the patient at the onset without satisfactory results, so prednisone was then prescribed and the symptoms were relieved soon after. After the symptoms of acute gout subsided, the patient took allopurinol.

\section{Case 2}

The second case was a 24-year-old man with left back and hip pain for 4 months and intermittent fever for 3 months. The left back and hip pain was relieved after rest, but not after exercise. The patient had fever, defined as a temperature above $38.5^{\circ} \mathrm{C}$, with chills. In the course of this disease, he did not experience any oral ulcer, diarrhea, joint swelling pain, iritis, skin rash, abdominal pain, frequent urination urgency or urinary pain. His only medical history was gout for more than 3 years, but he did not receive regular treatment. Physical examination revealed subcutaneous nodules of the extensor surface of the right elbow. Patrick's test of the right elbow was positive. Laboratory examination results, including complete blood count, electrolytes, urea, coagulation profile and muscle enzymes, were within normal limits. UA was normal at $386 \mu \mathrm{mol} / \mathrm{L}$ (reference range: $110-444 \mu \mathrm{mol} / \mathrm{L})$, creatinine was high at $112 \mu \mathrm{mol} / \mathrm{L}$ (reference range: $30-110 \mu \mathrm{mol} / \mathrm{L}$ ) and C-reactive protein (CRP) was high at $64.8 \mathrm{mg} / \mathrm{L}$ (reference range: $0-8 \mathrm{mg} / \mathrm{L}$ ). Short tau inversion recovery (STIR) MRI showed multiple bone marrow lesions, which appeared as edema involving the left side of the sacrum and ilium. The corresponding T1-weighted MRI showed hypointense signal of the left side of the sacrum and ilium (Figure 4). CT of the SIJ showed bone erosion of the bilateral SIJ (Figure 5). Treatment with nonsteroidal antiinflammatory drugs and antibiotics showed unsatisfactory response. CT-guided sacroiliac biopsy showed that MSU crystals were positive by polarized microscopy, although histopathology of the puncture objects did not show tophus formation and infiltration of acute inflammatory cells, but the infiltration of chronic inflammatory cells, which ruled out septic arthritis. His final diagnosis was gouty arthritis, and treatment with febuxostat and loxoprofen sodium relieved the symptoms.

\section{Discussion}

Gout is a common metabolic disease. In recent years, there have been more and more young patients with metabolism 


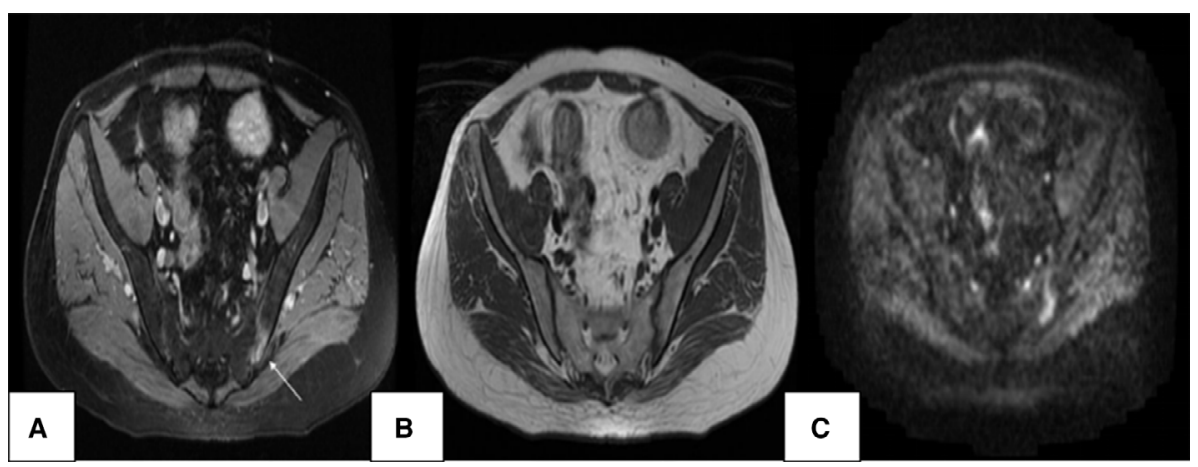

Figure 2 MRI of SIJ: (A) axial STIR sequence (TR 2500, TE 35) shows the hyperintense signal of left SIJ (arrow); (B) axial TI-weighted sequence (TR 450, TE 2 I) shows the hypointense signal of left SIJ; (C) DWI (TR 2000, TE 70) shows the hyperintense signal of left SIJ with edge blurring.

Abbreviations: DWI, diffusion-weighted imaging; MRI, magnetic resonance imaging; SIJ, sacroiliac joint; STIR, short tau inversion recovery; TR, repetition time; TE, echo time.

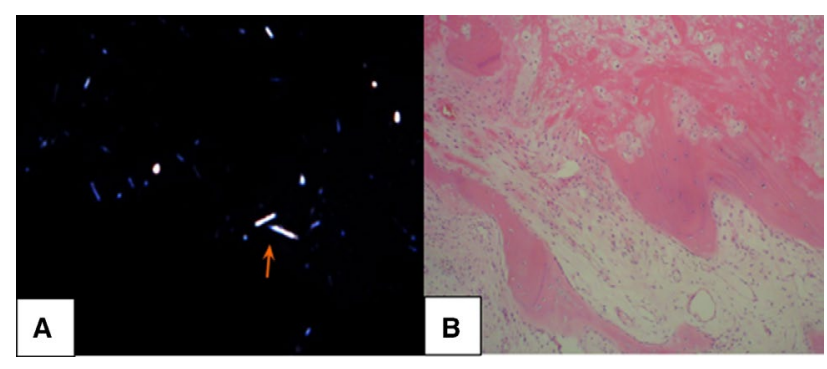

Figure 3 (A) Polarized microscopy revealed the urate acid crystals (arrow). (B) Histopathology of puncture objects did not show the tophus formation.

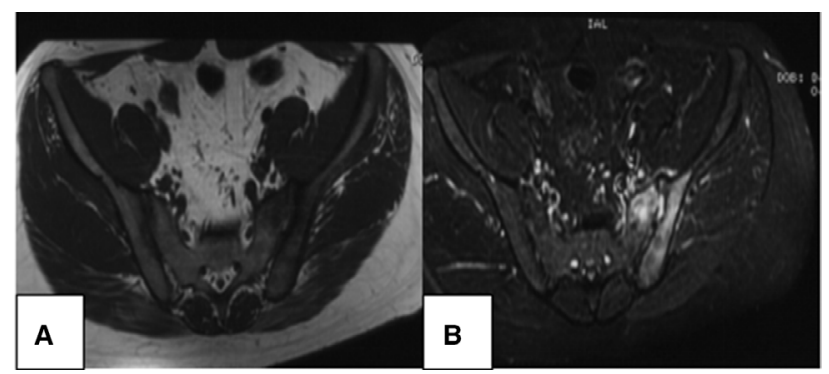

Figure 4 MRI of the SIJ: (A) axial TI-weighted sequence (TR 429, TE I2) shows the hypointense signal of the left side of the sacrum and ilium; (B) axial STIR sequence (TR 2400, TE 35): multiple bone marrow lesions, which appeared as edema involving the left side of the sacrum and ilium.

Abbreviations: MRI, magnetic resonance imaging; SIJ, sacroiliac joint; STIR, short tau inversion recovery; TR, repetition time; TE, echo time.

syndrome, hyperuricemia and gout due to change in lifestyle. The most common symptom is inflammatory monoarthritis due to the deposition of MSU crystals in the joints and connective tissue tophi, most often affecting middle-aged to elderly men and postmenopausal women. Acute gout is predominantly a disease of the lower extremities, but eventually involves the joints of any extremities, including the axial joint. In axial gout, any segment of the spine and its components (vertebral bodies, pedicles, lamina, ligaments, interapophyseal cartilage, epidural and intradural spaces)

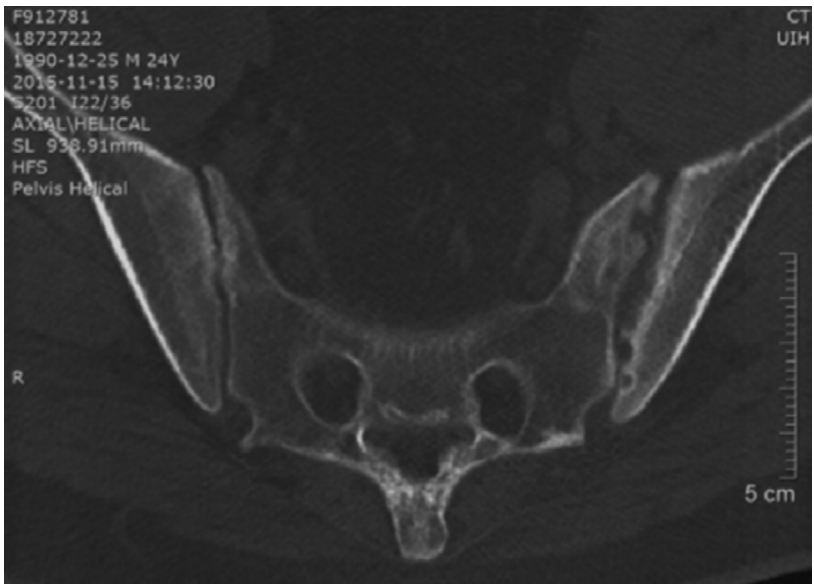

Figure $5 \mathrm{CT}$ of the SIJ $(120 \mathrm{kV}, 280 \mathrm{~mA})$ shows bone erosion of the bilateral SIJ and pseudo-widening of the left SIJ.

Abbreviations: CT, computed tomography; SIJ, sacroiliac joint.

and the SIJ may be involved, with lumbar involvement being the most common. ${ }^{1}$

The occurrence of gout in the SIJ has been reported in only a few cases. The incidence of SIJ involvement in chronic gout ranges from $7 \%$ to $17 \%$, based on radiographic findings. Clinical manifestations are nonspecific, such as low back and hip pain. ${ }^{2-4}$ Therefore, a patient with sacroiliac gout has symptoms which mimic the infection, SpA or osteoarthritis. $\mathrm{SpA}$ is probably the first diagnosis evoked in the case of chronic inflammatory back pain occurring before the age of 40 years. The prevalence of ankylosing spondylitis in the general population is linked to that of HLA-B27 and occurs most frequently in HLA-B27 young males. Therefore, two patients, in the current paper, were initially diagnosed as having SpA. Especially, the case 2 was misdiagnosed as the infection, who had the fever and unilateral side bone marrow edema in SIJ MRI, but this patient had no response to anti-infection therapy. Eventually, the diagnoses of SpA and infection were excluded through the pathological biopsy. 
Imaging examination of axial gout is nonspecific. CT is preferred over conventional radiography (CR) and MRI to identify axial gout. Compared with low sensitivity CR, CT can detect the subcutaneous and deep tissue tophi and show the intra-articular and juxta-articular erosion with sclerotic margins, but is unable to directly visualize the MSU crystal deposition. MRI can detect subcutaneous and deep tissue tophi, but the appearances are nonspecific. Dual-energy CT (DECT) can directly visualize the MSU crystal deposition, but sensitivity in early gout is not established. ${ }^{5-7}$

Two cases, in the current paper, showed the uncommon presentation of tophaceous deposits in the SIJ. The definitive diagnosis of gout was possible only by inspecting the synovial fluid or tophaceous material, and demonstrating the characteristic MSU crystals. Patients with symptomatic gouty SIJ can have the low back and hip pain or fever associated with local and systemic inflammatory response. Because of the inflammation and radiological findings, SIJ gout is often misdiagnosed as SpA or epidural abscess. ${ }^{8}$

Gout is known as a "great mimicker" with a diagnostic challenge. The ultimate diagnosis of tophaceous gout is histological, and CT-guided needle biopsy may be performed to avoid the surgical risks, although the sensitivity is limited. Two cases in the current paper underwent CT-guided needle biopsy in the SIJ. Histopathology of the puncture objects did not show the tophus formation, but polarized microscopy revealed the MSU crystals.

Previous literature on axial gout has been limited to case reports and case series, and very few cases on gouty sacroiliitis have been found during literature review. MSU crystals were identified in CT-guided aspiration of the SIJ. It is important to obtain the patient's disease history and perform a complete physical examination to decide whether the diagnosis of sacroiliitis (or any other musculoskeletal disorder) can be made. Gout can have various less typical forms. Gouty arthritis rarely involves the SIJ, but $\mathrm{SpA}$ is characterized by sacroiliitis. Approximately 46-73\% of gouty arthritis cases initially involve the first MTPJ followed by the tarsal joints, ankles, knees, fingers, wrists and elbows, but rarely the shoulders, hips, spine and SIJ. Acute gouty sacroiliitis should be included in the differential diagnosis of unilateral sacroiliitis in patients with a long-standing history of gout.

\section{Acknowledgments}

This work was supported by Hainan Medical Scientific Research Project (15320244A2002). The authors are grateful to all participants for their participation in this paper.

\section{Disclosure}

The authors report no conflicts of interest in this work.

\section{References}

1. de Mello FM, Helito PV, Bordalo-Rodrigues M, Fuller R, Halpern AS. Axial gout is frequently associated with the presence of current tophi, although not with spinal symptoms. Spine (Phila Pa 1976). 2014;39(25):E1531-E1536.

2. Konatalapalli RM, Demarco PJ, Jelinek JS, et al. Gout in the axial skeleton. J Rheumatol. 2009;36(3):609-613.

3. Malawista SE, Seegmiller JE, Hathaway BE, Sokoloff L. Sacroiliac gout. JAMA. 1965;194(9):954-956.

4. Alarcón-Segovia DA, Cetina JA, Díaz-Jouanen E. Sacroiliac joints in primary gout. Clinical and roentgenographic study of 143 patients. $A m$ J Roentgenol Radium Ther Nucl Med. 1973;118(2):438-443.

5. Lumezanu E, Konatalapalli R, Weinstein A. Axial (spinal) gout. Curr Rheumatol Rep. 2012;14(2):161-164.

6. Konatalapalli RM, Lumezanu E, Jelinek JS, Murphey MD, Wang H, Weinstein A. Correlates of axial gout: a cross-sectional study. J Rheumatol. 2012;39(7):1445-1449.

7. Dalbeth N, Doyle AJ. Imaging of gout-an overview. Best Pract Res Clin Rheumatol. 2012;26(6):823-838.

8. Dalbeth N, Doyle A, McQueen FM. Imaging in gout: insights into the pathological features of disease. Curr Opin Rheumatol. 2012;24(2):132-138.
Journal of Pain Research

Publish your work in this journal

The Journal of Pain Research is an international, peer reviewed, open access, online journal that welcomes laboratory and clinical findings in the fields of pain research and the prevention and management of pain. Original research, reviews, symposium reports, hypothesis formation and commentaries are all considered for publication.

\section{Dovepress}

The manuscript management system is completely online and includes a very quick and fair peer-review system, which is all easy to use. Visit http://www.dovepress.com/testimonials.php to read real quotes from published authors. 